\title{
Complete blood count-derived inflammatory markers in adolescents with primary arterial hypertension: a preliminary report
}

\author{
PIOTR SKRZYPCZYK', JOANNA PRZYCHODZIEN' ${ }^{1}$, MARTA BOMBIŃSKA2, \\ ZUZANNA KACZMARSKA ${ }^{2}$, MAGDALENA MAZUR ${ }^{2}$, MAEGORZATA PAŃCZYK-TOMASZEWSKA ${ }^{l}$ \\ ${ }^{1}$ Department of Pediatrics and Nephrology, Medical University of Warsaw, Warsaw, Poland \\ ${ }^{2}$ Student Scientific Group at the Department of Pediatrics and Nephrology, Medical University of Warsaw, Warsaw, Poland
}

\begin{abstract}
Aim of the study: The aim of our study was to evaluate selected inflammatory markers in children with untreated primary hypertension and to establish the relation between inflammatory markers and 24-hour ambulatory blood pressure monitoring (ABPM) and clinical and biochemical parameters.

Material and methods: In 54 children (15.12 \pm 2.02 years) with untreated primary hypertension, with excluded overt inflammation, we evaluated: neutrophils (NEU; 1000/ $\mu$ l), lymphocytes $(L Y M ; 1000 / \mu l)$, platelets $(P L T ; 1000 / \mu l)$, mean platelet volume (MPV; fl), neutrophil-to-lymphocyte ratio $(N L R)$, platelet-to-lymphocyte ratio (PLR), ABPM (OSCAR 2 SUNTECH), and selected clinical and biochemical parameters. The control group consisted of 20 healthy children (15.55 \pm 2.27 years).

Results: Children with primary hypertension had $(p<0.01)$ higher 24-hour systolic, diastolic and mean blood pressure, systolic and diastolic blood pressure loads, and pulse pressure. Hypertensive children did not differ in inflammatory indicators (NEU, LYM, PLT, MPV, NLR, PLR) from the control group. In 54 hypertensive children we found the following correlations: between office systolic and diastolic blood pressure and MPV $(r=0.35, p=0.011, r=0.36, p=0.008)$, between 24-hour ambulatory mean arterial pressure Z-score and $N L R(r=0.30, p=0.030), 24$-hour systolic blood pressure load and NLR $(r=0.38, p=0.005)$, plasma renin activity and neutrophil count, NLR, PLR $(r=0.47$, $p=0.016, r=0.64, p<0.001, r=0.42, p=0.033)$, urinary albumin loss and neutrophil count, NLR $(r=0.46, p=0.001$ and $r=0.42, p=0.003)$. Multivariate analysis revealed that office SBP Z-score was related to $M P V(\beta=0.35, p=0.008)$ and albuminuria to neutrophil count $(\beta=0.62, p=0.018)$.

Conclusions: In children with primary arterial hypertension there may be a relation between blood pressure, urinary albumin loss, and subclinical inflammation.
\end{abstract}

Key words: inflammation, adolescents, primary hypertension, complete blood count.

(Centr Eur J Immunol 2018; 43 (4): 434-441)

\section{Introduction}

Primary hypertension emerges from a complex interplay of genetic, environmental, and behavioral factors [1]. Due to rapid negative changes in children's life-style and diet, growing prevalence of primary hypertension in the pediatric population has been noted in recent studies. A multicenter American study revealed that essential hypertension was present in as many as $43 \%$ of children with elevated blood pressure and was the most common form of hypertension even in 7-year-old children [2].

The diagnosis of arterial hypertension, though clearly defined in international guidelines, is sometimes challenging in everyday clinical practice due to common prevalence of the white-coat phenomenon in pediatric patients.
Twenty-four hour ambulatory blood pressure monitoring (ABPM) allows earlier detection of abnormal blood pressure values and shows a better correlation with target organ damage when compared to office measurements [3]. Moreover, increased pulse pressure, high blood pressure variability and disturbed circadian blood pressure rhythm were found to be risk factors for the development of target organ damage, including left ventricular hypertrophy [4-6].

Studies of the last few years indicate the significant role of subclinical, low-grade inflammation in the pathogenesis of essential hypertension and target organ damage in both adults [7-9] and children [10]. Neutrophil count, neutrophil-to-lymphocyte ratio, platelet count, platelet-to-lymphocyte ratio, as well as mean platelet volume

Correspondence: Piotr Skrzypczyk, MD, PhD, Department of Pediatrics and Nephrology, Medical University of Warsaw,

63A Żwirki i Wigury St., 02-091 Warsaw, Poland, e-mail: pskrzypczyk@wum.edu.pl

Submitted: 7.08.2017; Accepted: 7.09.2018 
(MPV) were proposed as easily accessible, simple, and repeatable inflammatory markers associated with cardiovascular diseases such as acute coronary syndromes [11, 12], heart failure [13], and hypertension [14-16]. Preliminary experimental and clinical data suggest that the renin-angiotensin-aldosterone system (RAAS) might be the pathophysiological link between the immune system and cardiovascular burden $[17,18]$.

The aim of our study was to evaluate complete blood count (CBC)-derived inflammatory markers in children with untreated primary hypertension and to establish the relation between these inflammatory markers and 24-hour ambulatory blood pressure monitoring and clinical and biochemical parameters including plasma renin activity and aldosterone level.

\section{Material and methods}

We studied retrospectively 54 adolescents (37 boys, 17 girls) aged from 10.92 to 17.92 years with untreated primary hypertension hospitalized in our department in the years 2014-2017.

In all the studied children secondary forms of arterial hypertension were excluded by thorough medical history, physical examination and accessory examinations according to the European Society of Hypertension guidelines $[19,20]$. Also, based on medical history, physical examination, and evaluation of serum low resolution CRP (C-reactive protein) active inflammation (including common respiratory tract infection) was excluded in all studied children with arterial hypertension.

In all analyzed children we assessed the following clinical parameters: age (years), sex, duration of arterial hypertension (months), height (cm), weight $(\mathrm{kg})$, and body mass index (BMI; $\mathrm{kg} / \mathrm{m}^{2}$ ). Anthropometric variables were compared with Polish normative data and expressed as the Z-score [21]. Overweight and obesity were defined according to World Health Organization definitions as BMI Z-score values $>1$ and $>2$, respectively.

Office oscillometric blood pressure measurement was performed using Welch Allyn Vital Signs Monitor 300 (Welch Allyn Inc., Skaneateles Falls, NY, USA) according to European recommendations [19, 20]. Obtained values were expressed as $\mathrm{mm} \mathrm{Hg}$ and were compared with normative values [22] and expressed as the $\mathrm{Z}$-score.

In all children 24-hour blood pressure measurement was performed using a SUNTECH OSCAR 2 device (SunTech Medical, Inc., Morrisville, NC, USA) according to the American Heart Association guidelines [3]. Monitors were programmed to measure blood pressure every 15 minutes from $6 \mathrm{AM}$ to $10 \mathrm{PM}$ and every 30 minutes from $10 \mathrm{PM}$ to 6 AM. Periods of night-time rest and daytime activity were determined individually by the patients by providing relevant data in a diary. Data were compared to the reference values by Wühl et al. [23] and interpreted in accordance with the American Heart Association guidelines [3]. We analyzed only recordings with a sufficient number of successful blood pressure measurements (i.e. at least 40 measurements during 24 hours, 30 measurements during daytime activity, 10 measurements during nighttime rest, and at least one measurement during each hour [3]).

The following parameters were evaluated based on ABPM: systolic, diastolic and mean blood pressure (SBP, DBP, MAP) during 24 hours ( $\mathrm{mm} \mathrm{Hg}$ ), systolic and diastolic blood pressure load during 24 hours (\%), pulse pressure (mm Hg), nocturnal blood pressure dip (\%), and blood pressure variability.

Hypertension was defined as systolic and/or diastolic pressure $\geq 95^{\text {th }}$ percentile for sex, age, and height during 24 hours according to AHA guidelines [3]. In all the patients a Z-score value of mean arterial blood pressure during 24 hours was calculated using pediatric reference values [23]. Blood pressure load was calculated as a percentage of measurements $\geq 95^{\text {th }}$ percentile during 24 hours. Increased blood pressure load was defined as $>25 \%$ [3]. Pulse pressure was calculated as the difference between mean 24-hour systolic and diastolic blood pressure (PP/24 h) (mm Hg). Nocturnal systolic and diastolic blood pressure dip (SBP DIP and DBP DIP, respectively) was calculated as the difference between daytime pressure and nighttime pressure expressed as a percentage of the day value. Disturbed circadian blood pressure rhythm was defined as a nocturnal systolic or diastolic blood pressure dip less than $10 \%$. Disturbed circadian blood pressure rhythm was defined as a nocturnal systolic or diastolic blood pressure dip less than $10 \%$ [3]. Blood pressure variability was calculated as the standard deviation (SD) from the mean of all systolic or diastolic blood pressure values over 24 hours (SD SBP/24 h, SD DBP/24 h) [5].

In all the studied children peripheral automated complete blood count was performed using a Coulter LH 780 hematologic analyzer (Beckman Coulter Inc., Brea, CA, USA). The following complete blood count-derived inflammatory markers were analyzed: direct numbers of neu-

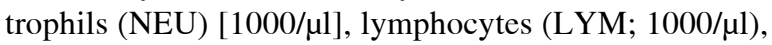
and platelets (PLT; 1000/ $\mu \mathrm{l})$, mean platelet volume (MPV; fl), and neutrophil-to-lymphocyte and platelet-to-lymphocyte ratios (NLR and PLR, respectively).

In addition, the following biochemical parameters were analyzed: glomerular filtration rate (GFR) according to the Schwartz formula $\left(\mathrm{ml} / \mathrm{min} / 1.73 \mathrm{~m}^{2}\right)$ [24], uric acid $(\mathrm{mg} / \mathrm{dl})$, cholesterol $(\mathrm{mg} / \mathrm{dl})$, triglycerides $(\mathrm{mg} / \mathrm{dl})$, and daily urinary albumin loss (mg/24 h). In 26 (48.1\%) hypertensive patients plasma renin activity (PRA) $(\mathrm{ng} / \mathrm{ml} / \mathrm{h}$ ) and aldosterone (ALDO) serum concentration ( $\mathrm{ng} / \mathrm{dl}$ ) in a standing position after 2 hours of walking were evaluated. Reference values for PRA and ALDO were as follows: PRA: 1.5-5.7 (ng/ml/h), ALDO: 2.52-39.2 (ng/ dl). Microalbuminuria was defined as daily albumin loss $>30(\mathrm{mg} / 24 \mathrm{~h})$ [20]. 
The control group consisted of 20 healthy children (12 boys, 8 girls) in whom all above-mentioned parameters except for plasma renin activity and aldosterone level were evaluated.

Low-sensitivity C-reactive protein was measured in the studied children using the VITROS 5600 Integrated System and was within normal limits $(<1.0 \mathrm{mg} / \mathrm{dl})$ in all the participants from the study and control groups.

The research project was approved by the local Ethics Committee (approval no. KB/58/2016). All procedures were performed in accordance with the Declaration of Helsinki on the treatment of human subjects. Informed consent was obtained from all participants $(\geq 16$ years) and their representatives included in the study.

Statistical analysis was conducted using the Dell Statistica 13.0 package (Dell Inc., Aliso Viejo, CA, USA). Normal distribution of variables was evaluated using the Shapiro-Wilk test. Regardless of distribution, data were presented as mean values \pm standard deviation. Differences between mean values of normally distributed variables were evaluated using the Student $t$ test for independent samples, and differences between mean values of non-normally distributed variables were evaluated using the Mann-Whitney U test. Frequencies were compared between groups using the chi-squared test and the exact Fisher test. Correlations between variables were evaluated using the Pearson linear correlation and Spearman's rank correlation, when appropriate. Variables related to blood pressure Z-scores, albuminuria and plasma renin activity with $p<0.200$ were analyzed by multivariate analysis using a generalized regression model. $p<0.05$ was considered statistically significant.

\section{Results}

Clinical and biochemical parameters in the study and control groups are presented in Table 1 . The groups did not differ significantly in terms of age and sex, but both BMI and BMI Z-score were significantly higher in children with hypertension. Office systolic and diastolic blood pressure were significantly higher in hypertensive patients. Among hypertensive patients $25(46.3 \%)$ were overweight and $8(14.8 \%)$ were obese, whereas there where $5(25 \%)$ overweight children in the control group. All patients in both groups had normal kidney function. We found no differences in lipids and uric acid levels between the groups. Six $(11.1 \%)$ children in the study group and no patients in the control group had microalbuminuria.

In the study group plasma renin activity and aldosterone level were as follows: PRA: $8.31 \pm 7.61 \mathrm{ng} / \mathrm{ml} / \mathrm{h}$, and ALDO: $86.69 \pm 123.72 \mathrm{ng} / \mathrm{ml}$, respectively.

The results of ambulatory blood pressure monitoring are shown in Table 2. Analysis of ABPM data showed that patients with hypertension were characterized by significantly higher systolic, diastolic and mean blood pressure, blood pressure load, and pulse pressure compared to the control group. The two groups did not differ in regard to blood pressure variability and nocturnal blood pressure dip.

Inflammatory markers derived from peripheral complete blood count are depicted in Table 3. Hypertensive patients were characterized by higher neutrophil and lymphocyte count, higher neutrophil-to-lymphocyte ratio, and lower platelet-to-lymphocyte ratio, though these differences did not reach statistical significance.

Table 1. Clinical and biochemical parameters in the study and control groups

\begin{tabular}{lccc}
\hline Parameter & Study group & Control group & $\boldsymbol{p}$ \\
\hline Number of patients $(n)$ & 54 & 20 & - \\
\hline Age (years) & $15.12 \pm 2.02$ & $15.55 \pm 2.27$ & NS $(0.431)$ \\
\hline Gender (boys/girls) & $37 / 17$ & $12 / 8$ & NS $(0.583)$ \\
\hline BMI $\left(\mathrm{kg} / \mathrm{m}^{2}\right)$ & $25.55 \pm 4.60$ & $22.05 \pm 2.22$ & 0.002 \\
\hline BMI Z-score & $1.20 \pm 0.75$ & $0.46 \pm 0.72$ & $<0.001$ \\
\hline SBP $(\mathrm{mm} \mathrm{Hg})$ & $139.57 \pm 10.35$ & $120.22 \pm 10.15$ & $<0.001$ \\
\hline SBP Z-score & $2.26 \pm 0.96$ & $0.55 \pm 0.98$ & $<0.001$ \\
\hline DBP $(\mathrm{mm} \mathrm{Hg})$ & $82.75 \pm 9.98$ & $73.06 \pm 7.23$ & $<0.001$ \\
\hline DBP Z-score & $1.41 \pm 0.81$ & $0.49 \pm 0.79$ & $<0.001$ \\
\hline GFRS $\left(\mathrm{ml} / \mathrm{min} / 1.73 \mathrm{~m}{ }^{2}\right)$ & $95.57 \pm 18.22$ & $97.84 \pm 16.84$ & NS $(0.629)$ \\
\hline Total cholesterol $(\mathrm{mg} / \mathrm{dl})$ & $160.92 \pm 31.12$ & $149.08 \pm 28.42$ & NS $(0.218)$ \\
\hline Triglycerides $(\mathrm{mg} / \mathrm{dl})$ & $93.76 \pm 39.44$ & $93.25 \pm 41.48$ & NS $(0.968)$ \\
\hline Uric acid $(\mathrm{mg} / \mathrm{dl})$ & $5.56 \pm 1.09$ & $5.40 \pm 0.89$ & NS $(0.578)$ \\
\hline Urinary albumin loss $(\mathrm{mg} / 24 \mathrm{~h})$ & $18.14 \pm 36.45$ & $3.83 \pm 1.67$ & NS $(0.201)$
\end{tabular}

BMI - body mass index, SBP - systolic blood pressure, DBP - diastolic blood pressure, GFRS - glomerular filtration rate calculated according to revised Schwartz formula, NS - not significant 
Table 2. 24-hour ambulatory blood pressure monitoring parameters in children in the study and in the control group

\begin{tabular}{lccc}
\hline Parameter & $\begin{array}{c}\text { Study group } \\
(\boldsymbol{n}=\mathbf{5 4})\end{array}$ & $\begin{array}{c}\text { Control group } \\
(\boldsymbol{n}=\mathbf{2 0})\end{array}$ & $\boldsymbol{p}$ \\
\hline Mean 24-hour SBP $(\mathrm{mm} \mathrm{Hg})$ & $134.85 \pm 6.97$ & $119.85 \pm 8.66$ & $<0.001$ \\
\hline Mean 24-hour DBP $(\mathrm{mm} \mathrm{Hg})$ & $74.11 \pm 5.37$ & $65.55 \pm 4.48$ & $<0.001$ \\
\hline Mean 24-hour MAP (mm Hg) & $94.33 \pm 5.02$ & $83.70 \pm 5.58$ & $<0.001$ \\
\hline Mean 24-hour MAP Z-score & $1.81 \pm 0.87$ & $0.02 \pm 0.79$ & $<0.001$ \\
\hline Mean 24-hour SBP load (\%) & $58.07 \pm 18.75$ & $15.70 \pm 10.69$ & $<0.001$ \\
\hline Mean 24-hour DBP load $(\%)$ & $33.50 \pm 18.67$ & $9.40 \pm 6.87$ & $<0.001$ \\
\hline 24-hour PP (mm Hg) & $60.78 \pm 6.80$ & $54.20 \pm 5.92$ & $<0.001$ \\
\hline 24-hour SBP variability & $13.69 \pm 3.47$ & $12.12 \pm 2.69$ & NS $(0.072)$ \\
\hline 24-hour DBP variability & $11.72 \pm 2.41$ & $10.56 \pm 2.23$ & NS $(0.066)$ \\
\hline Mean 24-hour HR (bpm) & $80.04 \pm 9.78$ & $76.30 \pm 7.34$ & NS $(0.126)$ \\
\hline SBP dip (\%) & $11.65 \pm 5.55$ & $11.12 \pm 6.07$ & NS $(0.725)$ \\
\hline DBP dip (\%) & $18.34 \pm 8.39$ & $15.84 \pm 8.07$ & NS $(0.252)$ \\
\hline Disturbed circadian blood pressure rhythm $(n, \%)$ & $25(46.3)$ & $8(40)$ & NS $(0.793)$ \\
\hline SBP - systolic blood pressure, DBP - diastolic blood pressure, MAP- mean arterial pressure, PP - pulse pressure, HR - heart rate, NS - not significant
\end{tabular}

Table 3. Inflammatory markers derived from complete blood count in children with primary hypertension and in the control group

\begin{tabular}{lccc}
\hline Parameter & $\begin{array}{c}\text { Study group } \\
(\boldsymbol{n}=\mathbf{5 4})\end{array}$ & $\begin{array}{c}\text { Control group } \\
(\boldsymbol{n}=\mathbf{2 0})\end{array}$ & $\boldsymbol{p}$ \\
\hline NEU $(1000 / \mu \mathrm{l})$ & $4.27 \pm 1.96$ & $3.59 \pm 1.16$ & NS $(0.150)$ \\
\hline LYM $(1000 / \mu \mathrm{l})$ & $2.22 \pm 0.56$ & $1.99 \pm 0.46$ & NS $(0.117)$ \\
\hline PLT $(1000 / \mu \mathrm{l})$ & $242.41 \pm 50.29$ & $245.15 \pm 55.82$ & NS $(0.840)$ \\
\hline MPV $(\mathrm{fl})$ & $9.10 \pm 1.40$ & $9.16 \pm 1.28$ & NS $(0.875)$ \\
\hline NLR & $2.06 \pm 1.30$ & $1.91 \pm 0.80$ & NS $(0.627)$ \\
\hline PLR & $114.73 \pm 32.96$ & $126.62 \pm 31.26$ & NS $(0.167)$
\end{tabular}

NEU - neutrophil count, LYM - lymphocyte count, PLT - platelet count, MPV - mean platelet volume, NLR - neutrophil-to-lymphocyte ratio, PLR - platelet-tolymphocyte ratio, NS - not significant
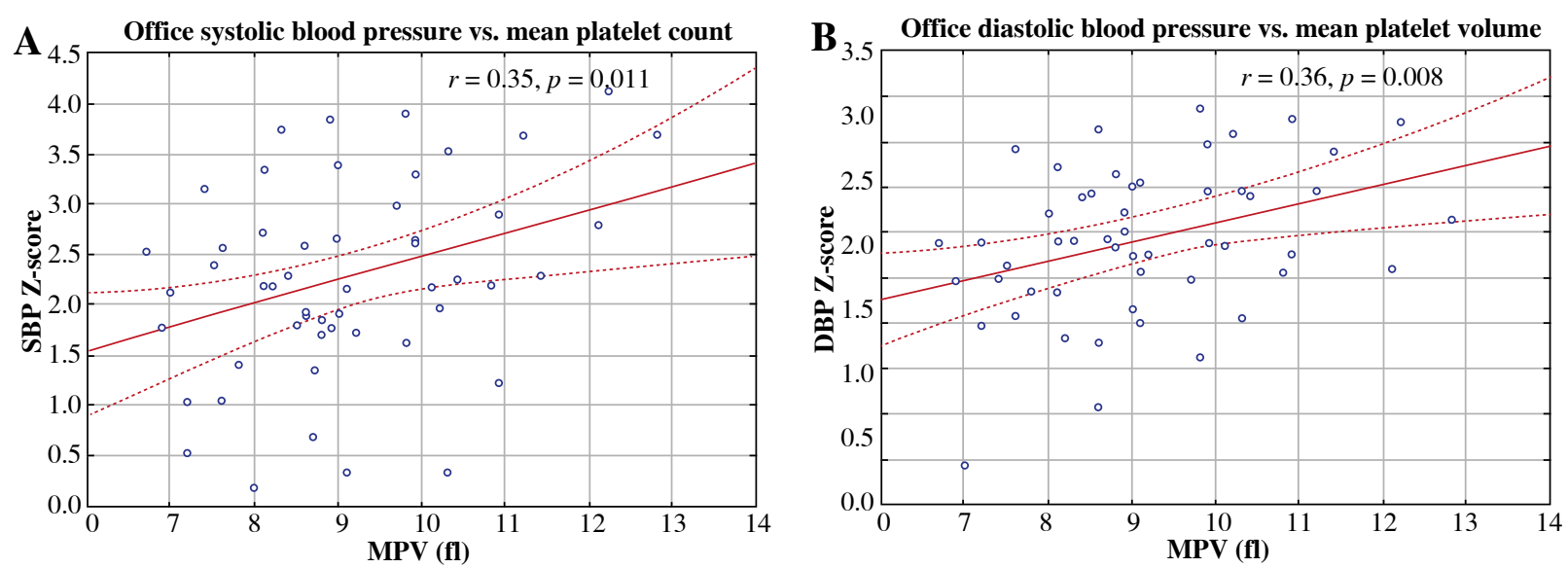

SBP - systolic blood pressure, DBP - diastolic blood pressure, MPV - mean platelet volume

Fig. 1. Correlations between mean platelet volume and office blood pressure in adolescents with primary hypertension 

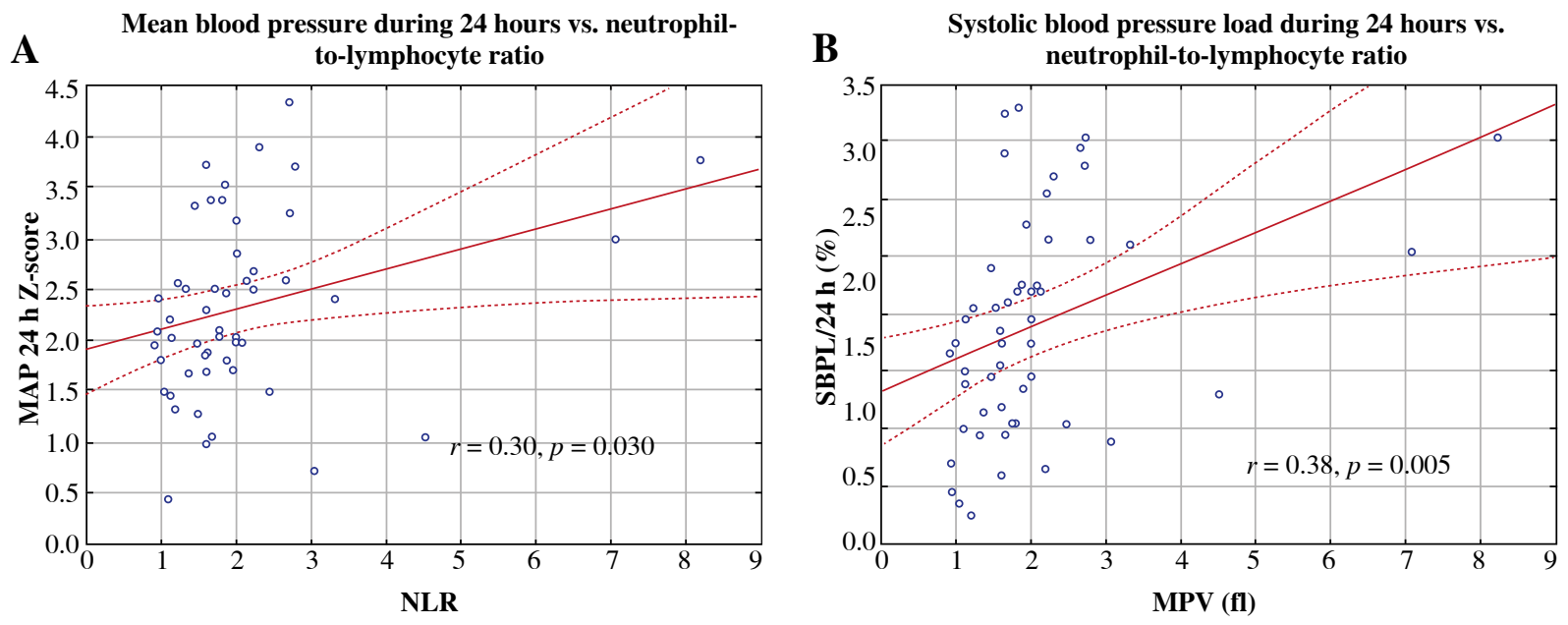

MAP $24 \mathrm{~h}$ - mean arterial pressure during 24 hours, SBPL/24 $\mathrm{h}$ - systolic blood pressure load during 24 hours, NLR - neutrophil-to-lymphocyte ratio

Fig. 2. Correlations between neutrophil-to-lymphocyte ratio and ambulatory blood pressure in adolescents with primary hypertension

In the whole group of 54 children with untreated primary hypertension we found positive correlations between mean platelet volume and office systolic blood pressure and diastolic blood pressure expressed as both $\mathrm{mm} \mathrm{Hg}$ and Z-scores (systolic blood pressure: $r=0.35, p=0.011$ and $r=0.35, p=0.011$, diastolic blood pressure: $r=0.36$, $p=0.007$ and $r=0.36, p=0.008$ ) (Fig. 1).

Neutrophil-to-lymphocyte ratio correlated positively with systolic blood pressure load during 24 hours $(r=0.38$, $p=0.005)$ and with mean arterial pressure Z-score during 24 hours $(r=0.30, p=0.030)$ (Fig. 2).

Neutrophil count, neutrophil-to-lymphocyte ratio and platelet-to-lymphocyte ratio correlated significantly with plasma renin activity $(r=0.47, p=0.016, r=0.64$, $p<0.001$, and $r=0.42, p=0.033$, respectively) (Fig. 3).

In addition, we found significant positive correlations between urinary albumin loss $(\mathrm{mg} / 24 \mathrm{~h})$ and neutrophil count and NLR $(r=0.46, p=0.001$ and $r=0.42, p=0.003)$ (Fig. 4). We did not find any significant correlations between analyzed inflammatory indicators and age except for a negative correlation of age and platelet count $(r=-0.39$, $p=0.003)$.

Multivariate analysis revealed that office SBP Z-score was related significantly to height $\mathrm{Z}$-score $(\beta=-0.31$, $p=0.019)$ and MPV $(\beta=0.35, p=0.008)$; albuminuria to neutrophil count $(\beta=0.62, p=0.018)$, and plasma renin activity to LDL cholesterol $(\beta=0.60, p=0.029)$. No significant determinants of MAP $24 \mathrm{~h}$ Z-score and office DBP $\mathrm{Z}$-score were found in multivariate analysis.

No significant correlations between analyzed inflammatory indicators and blood pressure were revealed in the control group.

\section{Discussion}

The results of our preliminary retrospective study showed that in children with primary hypertension office blood pressure values are related to mean platelet volume. Moreover, 24 hour mean blood pressure and 24 hour systolic blood pressure load are related to neutrophil-to-lymphocyte ratio. Our study might also suggest that activation of the renin-angiotensin-aldosterone system could be the link between inflammation and blood pressure elevation in these patients. Interestingly, neutrophil count and neutrophil-to-lymphocyte ratio were found to be positively related with degree of renal damage expressed as urinary albumin excretion in the study group. In contrast, no such dependences were found in the group of healthy children.

Neutrophils are the most abundant type of white blood cells and the first cells responding to inflammation, especially if it is caused by a bacterial infection, cancer or environmental exposure. They release many cytokines that trigger and amplify inflammatory reactions. Neutrophil-to-lymphocyte ratio (NLR), which can be derived from the white blood cell count, is an inexpensive, reproducible test and has shown up as a marker of systemic inflammatory response. Adult studies have shown that high NLR is associated with poor clinical outcomes in cardiac diseases [11-13, 16, 25]. While mainly high neutrophil count reflects inflammation, low lymphocyte count reflects poor general health and physiological stress [25]. Thus, blood NLR is an indicator of the overall inflammatory and stress status of the body. Our results indicate a pathophysiological relation between neutrophil-to-lymphocyte ratio, blood pressure and target organ damage also in pediatric 


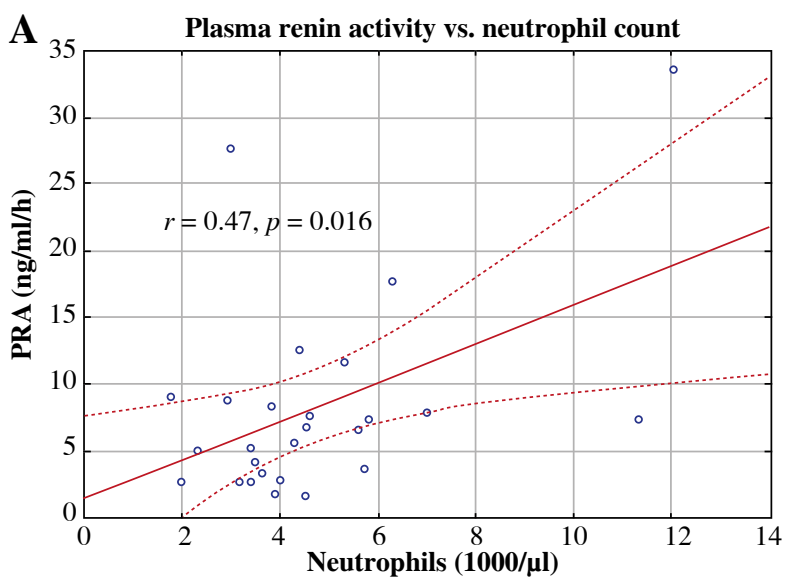

$\mathrm{C}_{35}$ Plasma renin activity vs. platelet-to-lymphocyte ratio

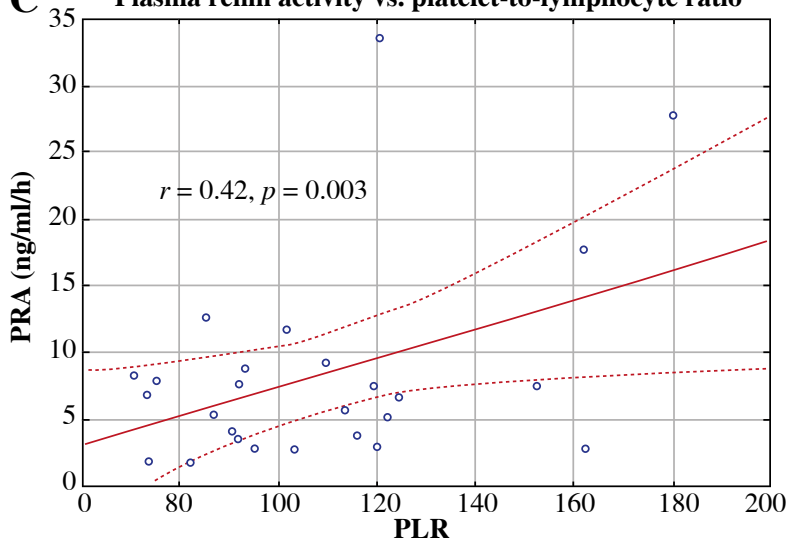

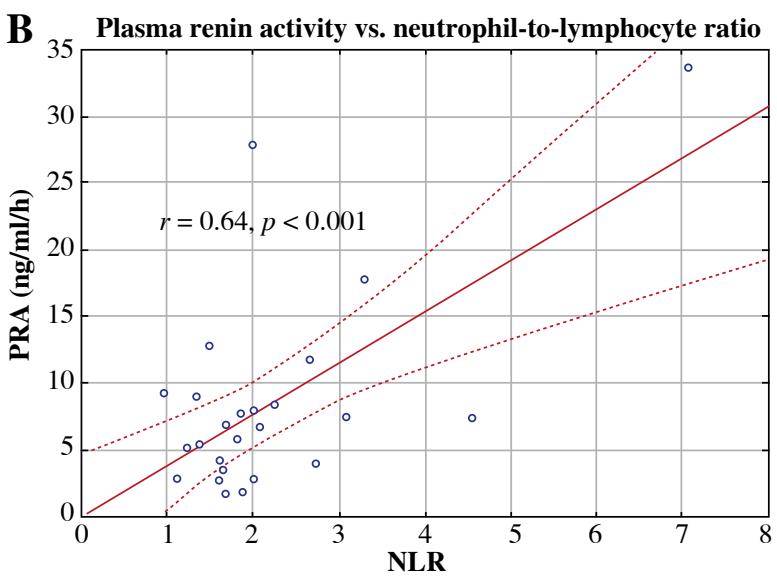

PRA - plasma renin activity, NLR - neutrophil-to-lymphocyte ratio, PLR - platelet-to-lymphocyte ratio

Fig. 3. Correlations between neutrophil count, neutrophil-to-lymphocyte ratio, platelet-to-lymphocyte ratio and plasma renin activity in adolescents with primary hypertension
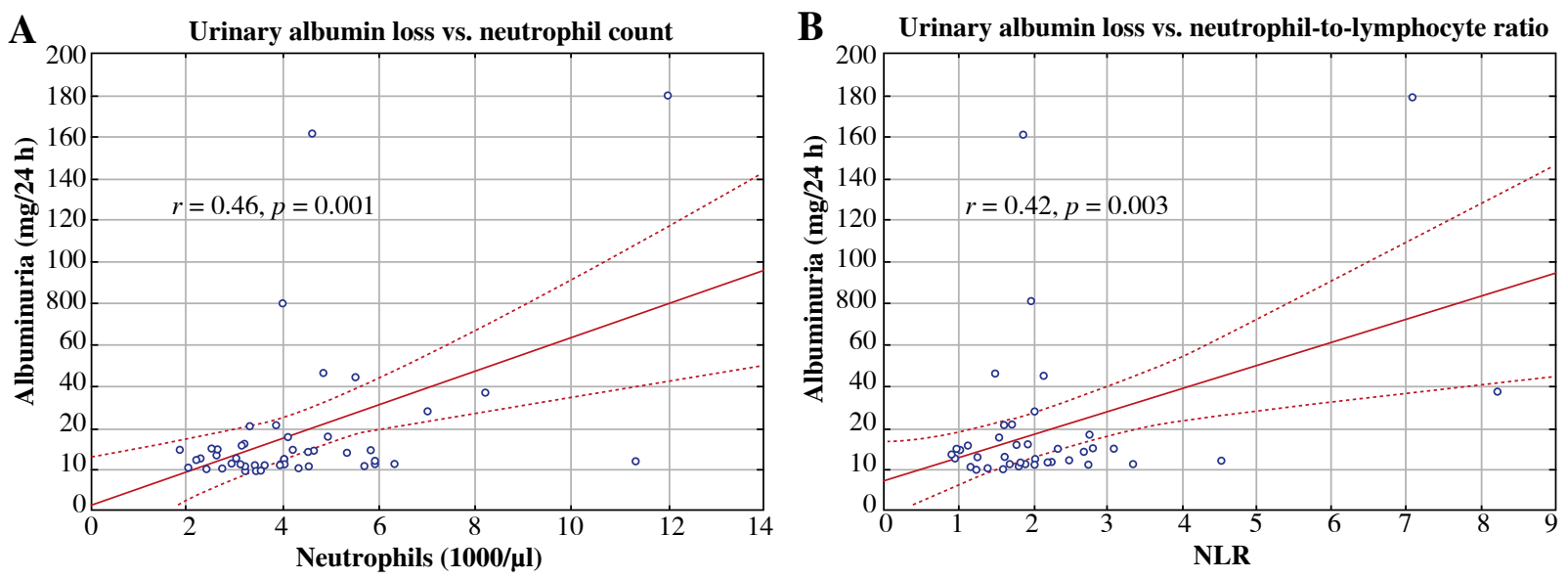

NLR - neutrophil-to-lymphocyte ratio

Fig. 4. Correlations between neutrophil count, neutrophil-to-lymphocyte ratio and urinary albumin loss in adolescents with primary hypertension

patients with primary hypertension. The positive relation between neutrophils and urinary albuminuria revealed in our pediatric group is in accordance with the results of Murai et al., who found that in adults urinary albumin is closely associated with low-grade inflammation evaluated as C-reactive protein level [26].
Although there is usually circadian variation in blood pressure (BP), such as more than a $10 \%$ decrease in systolic and diastolic BP during sleep compared to daytime (dipper), some normotensive and hypertensive subjects have no such circadian variation - they have been called non-dippers [3]. Adult patients with non-dipper hyperten- 
sion are associated with increased cardiovascular risk compared to patients with dipper hypertension [27]. Sunbul et al. found that adult patients with non-dipper hypertension had significantly higher NLR and PLR compared to dipper hypertension. Moreover, PLR more than 107 but not NLR was independent predictor of non-dipper status [16]. In our group of pediatric patients we did not find such a relation.

Large platelets are more active metabolically and enzymatically, and they have increased thrombotic potential in comparison to small platelets [28]. Mean platelet volume (MPV) has been shown to be a marker of platelet activation, which plays an important role in the pathophysiology of atherosclerosis [29]. Elevated MPV levels are thought to be closely associated with cardiovascular diseases such as acute coronary syndromes [28] and hypertension [14] (also masked hypertension [15]), though the pathophysiological mechanisms of elevated MPV in hypertension are not clearly understood. Interestingly, in our cohort MPV correlated positively with office systolic and diastolic blood pressure, whereas no such relation was revealed in ABPM and in healthy subjects. In addition, multivariate analysis found only a link between systolic blood pressure and platelet volume. Our results suggest a link between platelet activation and primary hypertension in adolescents, but the nature of this relation requires further investigations.

Nevertheless, it must be emphasized that clinical usefulness of CBC-derived inflammatory markers (MPV, NLR, PLR) in pediatric patients with primary hypertension still remains highly speculative. Notably, we have not found any significant differences in these indices between hypertensive and normotensive children, and many correlations found in univariate analysis were not significant in multivariate analysis. On the other hand, correlations between these markers and blood pressure were absent in healthy individuals.

Evidence from animal studies strongly supports an important role of inflammation and interleukin-6 (IL-6) in the pathogenesis of angiotensin II-mediated hypertension [17]. In addition, a few clinical studies have suggested that angiotensin receptor blockers (ARBs) have an anti-inflammatory effect that may provide additional benefits to patients beyond lowering of BP [30,31]. Chamarthi et al. found that hypertensive adults had higher baseline IL-6 and CRP compared with normotensives and IL-6 increased in response to ANGII in these patients. In the setting of RAAS activation by a low salt diet the authors demonstrated an independent positive association of PRA with CRP [32]. Interestingly, renin and angiotensin II are expressed in T-lymphocytes and this expression is related to subclinical inflammation [18]. Also, in our group of hypertensive children we revealed a significant positive relation between plasma renin activity and inflammatory indicators: neutrophil count, NLR, and PLR. We are deeply convinced that our preliminary results necessitate further studies on the mutual relation between the renin-angiotensin-aldosterone system and inflammation in hypertensive children.

The limitations of our study are the retrospective design and small number of patients, which could have influenced some of the results. Also the retrospective nature of our study precluded analysis of PRA and ALDO in all the patients from the study group and in the control group. We also did not evaluate hsCRP or cytokine concentration (e.g. IL-6 or TNF- $\alpha$ ), which are well-established and more precise markers of subclinical inflammation. In addition, evaluation of target organ damage was limited to basic tests assessing renal involvement (creatinine, urinary albumin loss). Further studies on larger patient groups, together with experimental studies, are necessary to reveal the link between primary arterial hypertension and the immune system.

\section{Conclusions}

In children with primary arterial hypertension there may be a relation between blood pressure, urinary albumin loss, and subclinical inflammation.

The authors declare no conflict of interest.

\section{References}

1. Litwin M, Feber J, Niemirska A, et al. (2016): Primary hypertension is a disease of premature vascular aging associated with neuro-immuno-metabolic abnormalities. Pediatr Nephrol 31: 185-194.

2. Gupta-Malhorta M, Banker A, Shete S, et al. (2015): Essential hypertension vs. secondary hypertension among children. Am J Hypertens 28: 73-80.

3. Flynn JT, Daniels SR, Hayman LL, et al. (2014): Update: ambulatory Blood Pressure Monitoring in Children and Adolescents: A Scientific Statement From the American Heart Association. Hypertension 63: 1116-1135.

4. Kawai T, Ohishi M, Kamide K et al. (2013): Differences between daytime and nighttime blood pressure variability regarding systemic atherosclerotic change and renal function. Hypertens Res 36: 232-239.

5. Sharma AP, Mohammed J, Thomas B et al. (2013): Nighttime blood pressure, systolic blood pressure variability, and left ventricular mass index in children with hypertension. Pediatr Nephrol 28: 1275-1282.

6. Litwin M, Niemirska A, Śladowska J, Antoniewicz J (2006): Left ventricular hypertrophy and arterial wall thickening in children with essential hypertension. Pediatr Nephrol 21: 811819.

7. Turak O, Ozcan F, Tok D, et al. (2013): Serum uric acid, inflammation, and nondipping circadian pattern in essential hypertension. J Clin Hypertens (Greenwich) 15: 7-13.

8. Bartczak D, Szymański Ł, Bodera P et al. (2016): Psychoneuroimmunological aspects of cardiovascular diseases: a preliminary report. Centr Eur J Immunol 41: 2019-216.

9. Trott DW, Harrison DG, et al. (2014): The immune system in hypertension. Adv Physiol Educ 38: 20-24. 
10. Litwin M, Michałkiewicz J, Niemirska A, et al. (2010): Inflammatory activation in children with primary hypertension. Pediatr Nephrol 25: 1711-1718.

11. Ozluk OA, Yilmaz M, Topal D et al. (2016): neutrophil to lymphocyte ratio is associated with proximal/middle segment of the LAD lesions in patients with ST segment elevation infarction. Centr Eur J Immunol 41: 386-391.

12. Tamhane UU, Aneja S, Montgomery D, et al. (2008): Association between admission neutrophil to lymphocyte ratio and outcomes in patients with acute coronary syndrome. Am J Cardiol 102: 653-657.

13. Uthamalingam S, Patvardhan EA, Subramanian S, et al. (2011): Utility of the neutrophil to lymphocyte ratio in predicting long-term outcomes in acute decompensated heart failure. Am J Cardiol 107: 433-438.

14. Nadar SK, Blann AD, Kamath S, et al. (2004): Platelet indexes in relation to target organ damage in high-risk hypertensive patients: a substudy of the Anglo-Scandinavian Cardiac Outcomes Trial (ASCOT). J Am Coll Cardiol 44: 415-422.

15. Guven A, Caliskan M, Ciftci O, et al. (2013): Increased platelet activation and inflammatory response in patients with masked hypertension. Blood Coagul Fibrynolysis 24: 170-174.

16. Sunbul M, Gerin F, Durmus E, et al. (2014): Neutrophil to lymphocyte and platelet to lymphocyte ratio in patients with dipper versus non-dipper hypertension. Clin Exp Hypertens 2014; 36: 217-221.

17. Brands MW, Banes-Berceli AK, Inscho EW, et al. (2010): Interleukin 6 knockout prevents angiotensin II hypertension: role of renal vasoconstriction and janus kinase 2/signal transducer and activator of transcription 3 activation. Hypertension 56: 879-884.

18. Coppo M, Bandinelli M, Berni A, et al. (2011): Ang II upregulation of the T-lymphocyte renin-angiotensin system is amplified by low-grade inflammation in human hypertension. Am J Hypertens 24: 716-723.

19. Lurbe E, Cifkova R, Cruickshank JK, et al. (2009): Management of high blood pressure in children and adolescents: recommendations of the European Society of Hypertension. J Hypertens 27: 1719-1742.

20. Lurbe E, Agabiti-Rosei E, Cruickshank JK, et al. (2016): 2016 European Society of Hypertension guidelines for the management of high blood pressure in children and adolescents. J Hypertens 34: 1887-1920.

21. Kułaga Z, Litwin M, Tkaczyk M, et al. (2011): Polish 2010 growth references for school-aged children and adolescents. Eur J Pediatr 170: 599-609.

22. Kułaga Z, Litwin M, Grajda A, et al. (2012): Oscillometric blood pressure percentiles for Polish normal-weight schoolaged children and adolescents. J Hypertens 30: 1942-1954.

23. Wühl E, Witte K, Soergel M, et al. (2002): Distribution of 24-h ambulatory blood pressure in children: normalized reference values and role of body dimensions. J Hypertens 20: 1995-2007.

24. Schwartz GJ, Muńoz A, Schneider MF, et al. (2009): New equations to estimate GFR in children with CKD. J Am Soc Nephrol 20: 629-637.

25. Gibson PH, Cuthbertson BH, Croal BL, et al. (2010): Usefulness of neutrophil/lymphocyte ratio as predictor of new-onset atrial fibrillation after coronary artery bypass grafting. Am J Cardiol 105:186-191.
26. Murai S, Tanaka S, Dohi Y, et al. (2014): The prevalence, characteristics, and clinical significance of abnormal albuminuria in patients with hypertension Sci Rep 4: 3884.

27. Cuspidi C, Sala C, Tadic M, et al. (2015): Non-dipping pattern and subclinical cardiac damage in untreated hypertension: a systematic review and meta-analysis of echocardiographic studies. Am J Hypertens 28: 1392-1402.

28. Chu SG, Becker RC, Berger PB, et al. (2010): Mean platelet volume as a predictor of cardiovascular risk: a systematic review and meta-analysis. J Thromb Haemost 8: 148-156.

29. Arslan N, Makay B, Hizli S, et al. (2013): Assessment of atherosclerosis in obese adolescents: positive correlation of mean platelet volume and carotid intima media thickness. J Paediatr Child Health 49: 963-968.

30. Pavlatou MG, Mastorakos G, Margeli A, et al. (2011): Angiotensin blockade in diabetic patients decreases insulin resistance-associated low-grade inflammation. Eur J Clin Invest 41: 652-658.

31. Fliser D, Buchholz K, Haller H (2004): Antiinflammatory effects of angiotensin II subtype 1 receptor blockade in hypertensive patients with microinflammation. Circulation 110: 1103-1107.

32. Chamarthi B, Williams GH, Ricchiuti V, et al. (2011): Inflammation and Hypertension: The Interplay of Interleukin-6, Dietary Sodium and the Renin-Angiotensin System in Humans Am J Hypertens 24: 1143-1148. 